\title{
Developing a Worksite-based Culturally Adapted Smoking Cessation Intervention for Male Hispanic/Latino Construction Workers
}

\author{
Noella A. Dietz, ${ }^{1,2}$ Taghrid Asfar, ${ }^{2}$ Alberto J. Caban-Martinez, ${ }^{2,3}$ Kenneth D. Ward, ${ }^{4}$ \\ Katerina Santiago, ${ }^{2}$ Estefania C. Ruano-Herreria, ${ }^{2}$ Laura A. McClure, ${ }^{3}$ and David J. Lee ${ }^{2,3}$ \\ 1 The Research Institute, Broward Health, Fort Lauderdale, FL \\ 2 Department of Public Health Sciences, University of Miami Miller School of Medicine, Miami, FL \\ 3 Sylvester Comprehensive Cancer Center, University of Miami Miller School of Medicine, Miami, FL \\ ${ }^{4}$ Division of Social and Behavioral Sciences, School of Public Health, University of Memphis, Memphis, TN
}

\begin{abstract}
ntroduction: Over 2.6 million Hispanic/Latino construction workers (CWs) live in the US; $91 \%$ of South Florida CWs are Hispanic/Latino. CWs have higher smoking and lower cessation rates than other workers. Limited access to cessation services, worksite turnover, and lack of interventions tailored to culture/occupation hinder cessation. Partnering with worksite food trucks to deliver unique cessation interventions may improve these efforts.

Aims: To explore a novel cessation approach, assess worker/worksite acceptability, and seek input into intervention development.

Methods: In 2016, we conducted five semi-structured focus groups with 37 smoking Hispanic/Latino CWs. Constant comparative analysis was used to examine a priori themes regarding smoking behaviours, cessation treatments, intervention delivery, cultural adaptation, and quit interest.

Results: CWs reported tremendous job stress. Most smoking occurred during the workday and most CWs did not use nicotine replacement therapy with past quit attempts. Most CWs were open to a worksite face-to-face group cessation intervention before work (many underutilize breaks and feel pressure to keep working). CWs felt it unnecessary to tailor the intervention to Hispanics/Latinos indicating smokers are the same regardless of race/ethnicity.

Conclusions: Findings demonstrate the need to consider work environments, job demands/stress, and worker preferences when developing accessible and acceptable cessation interventions.
\end{abstract}

\section{Introduction}

Annually, about 480,000 people in the US die from tobacco related illnesses (American Cancer Society, 2016; Centers for Disease Control and Prevention, 2016). Nationally, $15.1 \%$ of adults, 18 years and older, smoke cigarettes (Jamal et al., 2016); however, prevalence varies substantially by racial/ethnic sub-groups. Individuals self-identifying as non-Hispanic American Indians/Alaskan Natives have the highest smoking prevalence rate $(21.9 \%)$, while Hispanics/Latinos and nonHispanic Asians have the lowest $(10.1 \%$ and $7.0 \%$, respectively) (Jamal et al., 2015). However, within the US Hispanic/Latino population, smoking rates vary substantially by country of origin (Kaplan et al., 2014).
For example, a US population-based longitudinal study (2008-2011) showed 35\% of Puerto Rican men and 33\% of Puerto Rican women were current smokers and $31 \%$ of Cuban men and 22\% of Cuban women smoked (Kaplan et al., 2014). Puerto Rican and Cuban study participants were also the least likely to report successful quit attempts relative to other Hispanic/Latino subgroups (Merzel et al., 2015).

In the US, Hispanics/Latinos are the second fastest growing population, thereby making smoking among Hispanics/Latinos a growing public health concern (Brown, June 26, 2014; Martinez-Tyson, Pathak, SolerVila, \& Flores, 2009; Webb, Rodriguez-Esquivel, \& Baker, 2010). Further, relative to whites, Hispanics/Latinos are

Address for correspondence: Noella A. Dietz, PhD, Corporate Director of Research, Broward Health, Voluntary Associate Professor, UM Miller School of Medicine, 1600 S Andrews Avenue, Fort Lauderdale, FL, 33316. Email: Ndietz@browardhealth.org 
less likely to utilize nicotine replacement therapy (NRT), which can enhance quit rates by 50\%-70\% (Levinson, Perez-Stable, Espinoza, Flores, \& Byers, 2004; Stead et al., 2012). US Hispanic/Latino geographic settlement patterns show Florida ranking first, second, and third in the nation for the largest numbers of Cubans, Puerto Ricans, and Dominicans, respectively (Ennis, Rios-Vargas, \& Albert, 2011). Additionally, in Florida Hispanics/Latinos are $22.5 \%$ of the state's population (Ennis et al., 2011).

In Florida, many Hispanics/Latinos are employed in the construction sector (Caban-Martinez, Clarke, Davila, Fleming, \& Lee, 2011). Historically, blue collar workers, particularly construction workers (CWs), have higher smoking rates (39\%) than other occupational groups (Lee et al., October 2012; Lee, Fleming, Arheart et al., 2007). Furthermore, CWs are exposed to occupational hazards and toxins, like carbon monoxide from gasoline powered equipment and other air pollutants/aerosols working to increase a worker's risk for lung cancer and respiratory illnesses (Dong, Chowdhury, McCann, Trahan, \& Gittleman, 2002; Driscoll, Steenland, Imel Nelson, \& Leigh, 2004; Driscoll, Steenland, Prüss-Ustun, Imel Nelson, \& Leigh, 2004). Data from a previous study in MiamiDade County showed $91 \%$ of the construction workforce comprised Hispanic/Latino CWs; the study also showed over $54 \%$ of the workers were current smokers (CabanMartinez, Clarke, Davila, Fleming, \& Lee, 2011). CWs work mainly in outdoor areas where indoor smoking bans do not apply, are less likely to have health insurance, and are less likely to seek preventative healthcare services (Lee, Fleming, McCollister et al., 2007). Cessation efforts are hindered by factors like high jobsite mobility/turnover, limited access to cessation services, and the absence of cessation interventions targeting culture and work circumstances (Caban-Martinez, Clarke, Davila, Fleming, Lee, 2011; Hawkins, Kreuter, Resnicow, Fishbein, Dijkstra, 2008; Resnicow, Soler, Braithwaite, Ahluwalia, \& Butler, 2000; Sorensen, Barbeau, Hunt, Emmons, 2004; Sorensen, Barbeau, Stoddard et al., 2007).

One method to engage these smokers may be to partner with food trucks routinely visiting construction sites to support the delivery of a work-based smoking cessation intervention (see Caban-Martinez, Clarke, Davila, Fleming, \& Lee, 2011). Food truck visits temporarily draw CWs to a single construction site location, making behavioural intervention recruitment more efficient. Food truck operators could possibly carry health promotion materials too. Worksite-based food trucks may provide a novel tool where public health professionals can access and disseminate health education, including a culturally adapted work-based smoking cessation intervention. Therefore, we conducted this qualitative study to understand CW work environments with an aim to assess their acceptability of a culturally tailored smoking cessation intervention utilizing the food truck to facilitate smoking cessation.

\section{Methods}

\section{Design/Population}

To assess the practicality of this cessation method, we conducted focus groups exploring concepts around acceptability and sought input into intervention development. We conducted five focus groups $(n=37)$ over two months in 2016 investigating issues around tobacco use behaviours/norms with CWs to acquire feedback on the acceptability of using food trucks to support the delivery of a smoking cessation intervention targeting Hispanic/Latino male CWs. Participants were recruited through one large construction company with many sites and by the local safety manager. The local safety manager selected the worksites for participation in the focus groups based on how far along they were in the construction projects to ensure there would be an adequate number of workers at each site for recruitment. The local safety manager introduced the study at the sites to the employees during one of their regular morning meetings and said the study team would be on site to recruit for a research project. Study team members then went to the construction sites and approached CWs who smoked and asked them if they would like to participate in a focus group. To participate, respondents had to be 18 years or older, male Hispanic/Latino CWs, and selfreported current smokers, including light, and intermittent smokers. Whereas women have made gains in the construction industry, the occupation is still predominantly male (CPWR - The Center for Construction Research and Training, 2013).

\section{Focus Group Procedures}

After screening for eligibility, the research team explained the study to participants and subsequently consented them. With assistance from the site safety manager, focus groups were held on a large construction site in one of the office trailers in Miami-Dade County during lunch break and lasted 60 minutes. At the start of the focus group session, participants were given a short survey to be filled out anonymously so we could obtain basic demographic information, employment characteristics, and smoking behaviour data. Interview guides contained open- and closed- ended questions developed by investigators in tobacco control and occupational health, with expertise in CW populations. A priori topics included work environment (e.g., construction site environments, length of time on sites, smoking policies, hours of operation), tobacco use behaviours at home/work, knowledge, and previous use of smoking cessation treatments (e.g., NRT or other quit aids), preferred intervention delivery method (e.g., food truck, face-to-face sessions versus other modalities like computer-based), cultural issues regarding cessation, and quit interest (willingness to quit in the next month) (see Table 1).

Focus groups were moderated by two bilingual Hispanic/Latino public health graduate students with 


\section{Table 1}

Focus group guide

\begin{tabular}{|c|c|}
\hline Thematic Area & Focus Groups Questions \\
\hline $\begin{array}{l}\text { Workplace Background/ } \\
\text { Construction Sites }\end{array}$ & $\begin{array}{l}\text { - Tell me about the construction site that you work on. How long have you been on this site and for how much } \\
\text { longer do you expect to work here? } \\
\text { - When do you arrive at work? You know, are you right on time or do you get there early and hang out a little before } \\
\text { you have to clock in? } \\
\text { - What do you usually do when you get a break? Do you stay on site? Go off site? How do you occupy your time } \\
\text { when you have your break? I also understand that people sometimes skip their breaks. } \\
\text { - At the end of your shift, do you go home right away or do you hang out a little while and talk to other people that } \\
\text { you work with? } \\
\text { - What do you usually do for your meals or lunch? Use the lunch truck? Bring your own? Go off site? } \\
\text { - Does your workplace offer special programs for its workers like health information, or any other programs? I want } \\
\text { to know who provides your health and safety information. How does that work? What about any smoking } \\
\text { cessation programs? Would you like it if they did offer programs for you? Education programs or health or } \\
\text { whatever? } \\
\text { - At your worksite, are there rules about where you can and cannot smoke? What are they? Do people abide by } \\
\text { them? Do you see a lot of people smoking on the job site? Do a lot of your coworkers smoke? About how many? } \\
\text { Does it vary by the different job sites that you've worked? } \\
\text { - What about you? Do you smoke on the job site? Where do you smoke? Do you or the guys you work with smoke } \\
\text { during lunch breaks? Do you usually smoke with coworkers? On a break? } \\
\text { - Do coworkers look down at you if you smoke? } \\
\text { - Would you say that there are more people who smoke at work, say, compared to where you live? }\end{array}$ \\
\hline
\end{tabular}

Thoughts on Cessation Treatments and Interventions
Cessation Preferences for Hispanic/Latino Male Construction Workers and Openness to Quitting
- There are a lot of different ways to quit smoking that are helpful. Some people quit on their own and other people get help. The types of help that work are talking to a doctor or counsellor, using the State Quitline, and using medication such as nicotine patch or Chantix. Have you tried any of these things to help you quit? What was useful? If you decided to quit again, which of these might you give another try?

- Would you be willing to talk to someone to help you quit? If so, what kind of a person do you think would be helpful to you? For example, a doctor? A nurse or counsellor? A co-worker or friend who has been quit for a long time? A guy rather than a woman? Someone older than yourself? Who else?

- Would you want a face-to-face intervention? What about weekly meetings? Or would you prefer something on the telephone? What makes you want that type of intervention?

- If someone was helping you to quit smoking, how often would you want to talk to that person? Would you want reminders about not smoking or reminders to encourage you to stay smoke free? Like a telephone call or text message?

- Where would you like to have the intervention? Is this something that you want to do online, on the phone, or in person? Would you consider your workplace a suitable site to do an intervention? If someone could give you a quick one-on-one intervention at work would you be interested in quitting that way?

- Can you think of reasons that would make you stop using any of these quit aides to help you stop smoking? Like NRT gum or patches?

- Are there any reasons that you would not continue with a treatment or intervention for quitting smoking? For example, if you had to drive someplace to receive the intervention would that stop you from wanting to continue using it or if it was expensive... things like that. Can you think of things that might stop you from wanting to try that intervention?

- Are there issues that should be considered when trying to help Hispanic construction workers quit smoking? Are certain approaches more or less likely to work for them? Why?

- Are there any cultural issues that should be considered, such as acculturation, cultural values-family, collectivism, machismo (men can smoke but women cannot), living situations, etc.

- Would you want your work site to help you quit smoking (e.g., having them fax a referral for you to quit to the QuitLine, provide the service on site during your lunch/breakfast break, provide NRT for free)?

- How interested are you in quitting in the near future (within the next month)? If not very interested: what would have to happen to make you interested? What if we could offer you some help to quit smoking, would you like to try?

- Would you be interested in quitting smoking if we could do a smoking cessation intervention at your work where it would be one-on-one and it could be during your lunch break? Why? (Cost effective? Because they smoked they understand how it feels to quit? Why not? If you are not sure, what are you uncertain about?

- If we had someone trained who went around on a lunch truck, would that be an effective way to help you quit smoking? 
backgrounds in occupational health and clinical psychology. Moderators were debriefed after each focus group session to assess participant's interest and to probe for any incidences that may have occurred. Interviews were conducted in Spanish based on participant preference. Interviews were audiotaped and transcribed verbatim by a bilingual study team member. The university's Institutional Review Board approved the protocol. Respondents received a $\$ 25$ incentive for participating in the focus groups.

\section{Analysis}

Survey data were analysed using SPSS and descriptive statistics were used to describe the characteristics of the sample. Qualitative data were analysed using a general inductive analysis, the constant comparative method, allowing for the emergence of data themes (Corbin \& Strauss, 2007; Glaser \& Strauss, 1967; Thomas, 2006). Qualitative data analysis is an iterative process and the resulting concepts/themes are derived from the raw data so concepts and themes build upon one another (Corbin \& Strauss, 2007; Thomas, 2006). Using constant comparative analysis, focus group data were coded around several a priori and emergent themes until saturation was reached (Boeije, 2002). In other words, we examined items within each transcript within the same group, then we compared each transcript to one another across transcripts (data) to determine if the concepts or themes were present in each of the transcripts (Boeije, 2002; Pope, Ziebland, \& Mays, 2000; Hewitt-Taylor, 2001). Themes are recurring subject areas described by respondents where we examine responses that fit into each thematic area (Boeije, 2002). The study investigator reviewed each transcription as it was completed, discussed the initial findings with the moderators to ensure data accuracy, and reread the transcriptions for content (Kitto, Chesters, \& Grbich, 2008). The investigator identified a theme and compared it in each transcript for similarities or differences (Boeije, 2002; Hewitt-Taylor, 2001; Pope et al., 2000). The investigator met with the moderators to discuss and verify findings for quality control (Boeije, 2002; Kotto, Chesters, \& Grbich, 2008). The data were categorized under a priori theme labels, specifically smoking behaviours (tobacco use patterns), cessation treatments, and the development of the smoking cessation intervention (preferences that include a face-to-face group treatment and an intervention before work). We also identified two emergent contextual factors influencing CW participation in a work-based culturally adapted smoking cessation intervention; specifically, we identified the work environment and stress as contributors to tobacco use patterns and cessation.

\section{Results}

The short survey showed the majority of participants were between 36 and 55 years (54\%), had a high school degree or less $(62.1 \%)$, and earned $\$ 30,000$ or less $(70.3 \%)$ (Table 2$)$. CWs tended to work day shifts $(81.1 \%)$, were worried about employment (59.4\%), and worked on job sites where they were exposed to numerous pollutants (73\%). Further, $91.9 \%$ of CWs had smoked a cigarette in the past month, with most participants smoking every day $(62.2 \%)$. The number of years CWs smoked ranged from one to 30 , with only two participants smoking more than that. Finally, when we asked the number of cigarettes smoked per day, we saw a similar percentage of participants who were light, moderate, or heavy smokers (see Boulous et al., 2009). Despite efforts to recruit selfreported current smokers, as seen in the survey data, the focus groups contained a possible non-smoker.

The five focus groups ranged in size from four to 10 participants, with an average participant size of seven (see Table 2 for exact group sizes). During the focus groups, we asked participants information about construction sites, coworkers, and site smoking policies to have a fundamental understanding of the occupation and tobacco work rules. Based on the focus group responses, we found employment ranged from one day to three years, with a median of 4.5 months. CWs tended to move from site to site frequently, so length of stay on each site is variable; they work on a site until their part of the job is finished then move to the next site. Also, CWs tended to arrive at the worksite between 6:00-7:00 AM and stay on site until it was time to leave for the day. Worksites have smoking restrictions ranging from no smoking to designated smoking areas. For example, CWs are not allowed to smoke near flammable storage areas or woodpiles, but can smoke outside worksite fences. However, CWs routinely ignore the rules and smoke on site while working, on breaks, and in front of the site. When examining participant preferences for a work-based cessation intervention, we found two common themes associated with tobacco use behaviours and three main themes specific to the intervention.

\section{Tobacco Use Patterns}

We asked respondents about their smoking patterns on/off the worksite. For instance, we asked participants if they smoked at the worksite, during lunch/breaks, on the way home, or at home. The majority said they smoked at work, during lunch/breaks, and at home. However, most participants said they smoked more at work than elsewhere. For instance:

I smoke while working. (Group 1; CW4)

... it's something common, smoking at the job sites, it's something common.

$\mathrm{Me}$, if I am not smoking [at work] they put a cigarette in my mouth. (Group 1; CW4)

...I leave work and the weekends its worse, but during the week when I arrive at home, I smoke three or four cigarettes, no more; here at work, I smoke a lot more, 10 or 11 . (Group 3; CW5) 


\section{Table 2}

Sociodemographic characteristics, employment characteristics, and smoking behaviour for Hispanic/Latino male construction workers $(n=37)$

Focus Group Participant Characteristics $n(\%)$

Group Sizes

Group 1

$4(10.8 \%)$

Group 2

Group 3

Group 4

Group 5

Age

$18-24$

$6(16.2 \%)$

25-35

$36-45$

46-55

$56-65$

Refused

Marital Status

Single

Married/Partnered

Separated/Divorced

Hispanic/Latino Ancestry

Dominican

$2(5.4 \%)$

Central American

Cuban

$6(16.2 \%)$

$13(35.1 \%)$

Mexican

South American

$1(2.7 \%)$

$5(13.5 \%)$

Other

Refused

$1(2.7 \%)$

$9(24.3 \%)$

Education

Less than HS

High School Degree

Some College

$14(37.8 \%)$

$9(24.3 \%)$

$6(16.2 \%)$

Trade School/Vocational

College Graduate or More

$3(8.1 \%)$

$4(10.8 \%)$

Refused

$1(2.7 \%)$

Income Per Year

$\$ 30,000$ or less

Greater than $\$ 30,000$

Refused

$9(24.3 \%)$

$2(5.4 \%)$

Employment Schedule

Regular Daytime Shift

$30(81.1 \%)$

Rotating Shift

$1(8.1 \%)$

Other

$2(5.4 \%)$

Refused

$4(10.8 \%)$

\section{Table 2}

Continued

\begin{tabular}{lr}
\hline Focus Group Participant Characteristics & $n(\%)$ \\
\hline Worried about Employment & \\
Strongly Agree/Agree & $22(59.4 \%)$ \\
Strongly Disagree/Disagree & $11(29.7 \%)$ \\
Refused & $4(10.8 \%)$
\end{tabular}

Exposed to Vapours/Gas/Dust/Fumes at Work

$$
\begin{array}{lc}
\text { Yes } & 27(73.0 \%) \\
\text { No } & 7(18.9 \%) \\
\text { Refused } & 3(8.1 \%)
\end{array}
$$

Smoked 100 Cigarettes or More in Lifetime

$\begin{array}{lr}\text { Yes } & 29(78.4 \%) \\ \text { No } & 4(10.8 \%) \\ \text { Refused } & 4(10.8 \%)\end{array}$

Smoked a Cigarette in the Last 30 Days

Yes

$34(91.9 \%)$

No

Refused

$1(2.7 \%)$

Smoke Every Day, Some Days, or Not at All Every Day

$23(62.2 \%)$

Some Days

$12(32.4 \%)$

Not at All

$1(2.7 \%)$

Refused

$1(2.7 \%)$

Number of Years Smoked

$1-5$

$6(16.2 \%)$

6-10

$6(16.2 \%)$

11-15

$6(16.2 \%)$

$16-20$

$3(8.1 \%)$

21-30

$8(21.6 \%)$

$31+$

$2(5.4 \%)$

Refused

$6(16.2 \%)$

Number of Cigarettes Smoked Per Day

1-4 Cigarettes

$12(32.4 \%)$

5-10 Cigarettes

$10(27.0 \%)$

11-14 Cigarettes

$1(2.7 \%)$

15-19 Cigarettes

$3(8.1 \%)$

$20+$

$10(27.0 \%)$

Refused

$1(2.7 \%)$

Past Quit Attempts

$\begin{array}{lc}\text { Never } & 8(21.6 \%) \\ \text { One } & 2(5.4 \%) \\ \text { Two or More } & 24(64.8 \%) \\ \text { Refused } & 3(8.1 \%)\end{array}$




\begin{tabular}{lr}
\hline $\begin{array}{l}\text { Table } 2 \\
\text { Continued }\end{array}$ \\
\hline Focus Group Participant Characteristics & $n(\%)$ \\
\hline Use NRT w/ Past Quit & \\
Attempts & \\
Yes & $4(10.8 \%)$ \\
No & $26(70.3 \%)$ \\
Refused & $7(18.9 \%)$ \\
\hline
\end{tabular}

When I get home I don't smoke, I don't really smoke at home... [Question: Then number 2 can't smoke inside the house?] No. (Group 4; CW2)

$\mathrm{Me}$ ? If I don't work, I can stop smoking, if I don't work frequently I can... I stopped one year but when I work I smoke because I have many problems with my machines. (Group 1; CW4)

For CWs who smoked at home, they used cigarettes to relax after work. The relaxation process often included coffee or alcohol, acting as triggers to smoke. Respondents explained they watched television, drank coffee, or had an alcoholic beverage after work. For example:

I make coffee, I sit in front of the TV to watch the news, and I smoke my cigarette. (Group 2; CW3)

I drink a beer and I smoke a cigarette. (Group 2; CW9)

[The relationship between smoking and alcohol]... It's like a marriage. (Group 3; CW1)

\section{Knowledge and Previous Use of Cessation Treatments}

We asked respondents about cessation aids they previously tried. Only three respondents said they used a Federal Drug Administration (FDA) approved cessation product (i.e., prescription tablets, patches, and gum). One participant did not like his reaction to it and stopped, another switched to an alternative method, and one relapsed. Most respondents used either nothing or tried e-cigarettes to quit smoking. For instance:

When I arrived in the United States, they gave me pills, but I did not [take] them because they gave me a bad reaction... Dizzy... And after a year, I stopped smoking, but it was on my account, I was a year and two months without smoking. (Group 3; CW1)

When I want to stop smoking, I stop smoking and that is it... [Another respondent echoed this observation] Same with me, without gum or patches or anything. (Group 5; CWs5,6)

I tried the vapors, in the morning, in the morning my throat was too dry; I used it for two or three days and began again with the cigarettes. (Group 3; CW9)

...I tried an electronic cigarette and that did not work. (Group 3; CW6)

\section{Cessation Preferences}

We asked respondents open-ended and closed-ended questions about quitting smoking using various formats, including the food truck visiting construction sites several times a day, a face-to-face intervention at work, a home- based intervention, a computer-based intervention, etc. Moderators specifically asked if they would be interested in having the intervention at lunch hour and using the food truck. Two key themes emerged: the intervention preference was a face-to-face group treatment relative to other formats and the timing of the intervention that worked best for CWs was before work. Respondents preferred the face-to-face intervention because they felt it provided them with support and it encouraged their full participation. For example:

\footnotetext{
Face-to-face, not through the phone.... (Group 3; CW1) All support is good. (Group 2; CW6)

Because I see the person, I get more strength and more in the mood. (Group 2; CW5)

A counselor [to talk to someone to quit]... Face-to-face. (Group 5; CW2)

Face-to-face you're in contact, through the phone one can be doing something else, watching a baseball game and you have the television on, and you're not paying attention. (Group 2; CW9)
}

The CWs all commented on the high level of stress at the job site, which factored into why they preferred to receive the intervention before work and why they tended to smoke more at work than elsewhere. CWs were open to having the food truck carry NRT; however, they were not open to using the truck at lunch break. They felt the intervention needed to take place before/after work and the food truck could be used to carry NRT. Participants explained not everyone came to the food truck at lunchtime - it varied by where they were in their projects and whether or not they brought their own lunch. Many participants worked through lunch to get a project completed on time. Participants also worried that if the intervention took too long, they would lose pay for the time they were not working. For instance:

\footnotetext{
[Question: If the lunch truck brought the gum and patches to help stop smoking would you utilize it?] Yes. (Group 1; CW4)

[...but if the truck, even though your wife prepares your food, if the truck had the patches and gum, would you utilize it?] Yes I would utilize it. (Group 1; CW1)

I am not in agreement with the lunch truck [at lunch break], for me at least, when I am on my break I don't stop, I don't go down for lunch, I don't go down. (Group 3; CW7)

[The intervention]... It would be after work or before work. (Group 4; CW10)

It would be good if they could come here to our worksite since we are always here. (Group 5; CW2)

[...lunchtime is a good time for the intervention?] In the morning, before beginning the workday. (Group 5; CW4) Because we come to work and we have to work during the time they are paying us. An intervention is time lost for the company and they can take retaliation for that. (Group 5; CW4)
}

This also factored into participants' level of job stress. Participants explained they were under great stress to complete tasks on time. As several CWs stated when asked about stress, 
It would be good to receive information about stress, about how to deal with stress; sometimes when someone is stressed they smoke, but one shouldn't do that because it affects a little bit of your health... (Group 2; CW9)

Well you will have to talk to work, the wife, the problems with the kids, the bills, the stress that falls on you, and more if you come here you have to work to the maximum. (Group 4; CW11)

The pressure, the pressure to run our chores, we take care of 44 people, we have 44 headaches and it's very stressful. We were from the garage to the roof, and that is all day going up and down, going up and down, then it is a lot of stress from the time you arrive at work. The supervisors of [company name] call you when you arrive on the roof and they are on the bottom and then if they call you, you have to go down again to the garage. You have to attend to them, then this job is not like being in an office here, it's up and down everywhere. (Group 3; CW9)

Finally, we asked respondents how they felt about a smoking cessation intervention culturally adapted for Hispanics/Latinos to determine participant receptivity to having an intervention that was culturally specific. Respondents told us it was not necessary to adapt the intervention for ethnicity because everyone was the same. Respondents preferred an intervention that was not culturally adapted per se. We also asked if there were particular things that needed to be considered (e.g., familialism or language) when developing the intervention specifically for Hispanic/Latino CWs. For instance, current smokers stated that we are all the same race:

No, in my opinion, all of the races [are] the same... The color can change but the blood is the same. (Group 1; CW4)

No, the smokers are the same, here, in Africa, in China, or all over. (Group 3; CW7)

I think that smoking is a personal problem, it is not something that has to do with culture. (Group 5; CW4)

\section{Discussion}

Our data identified critical factors associated with developing a novel smoking cessation intervention targeting male Hispanic/Latino CWs. We prioritized the necessary elements to be included in the intervention to help this population of smokers quit (i.e., using NRT, going to the worksite where the CWs spend most of their time). We addressed the delivery method and timing acceptable for CWs on a busy construction worksite. Further, our data showed most respondents were not receptive to culturally tailoring the intervention.

Male CWs who currently smoke felt a face-to-face group cessation format at the construction site, compared to telephone- or computer-based formats, was more acceptable since it catered to where they spent most of their time, and encouraged participants to be present and attentive to the cessation message. Further, focus group participants were open to using the food truck to deliver NRT and support the intervention, but stated the intervention had to take place before work. In this instance, the food truck is secondary to the face-to-face intervention and is used as a means to gather the participants rather than the main delivery method of the intervention. Intervention delivery and timing are critical components of the intervention as CWs are not paid for the time they are not on the job and are fired from jobsites where they do not complete their daily job assignments.

We probed respondents' receptivity to a culturally specific intervention. Because participants felt all racial/ethnic groups are the same, they were not receptive to culturally adapting cessation delivery. Targeted communication often includes demographic characteristics, while tailored messaging is more customized to culture (Hawkins et al., 2008). While the focus group participants were open to having the focus groups targeted in their communication (i.e., Spanish language and location), participants were less open to tailored messaging for the intervention that would have been highly customized to Hispanics/Latinos (i.e., familialism, simpatia (sympathy), social support, etc.) (Hawkins et al., 2008). It is important to note, however, that these results are preliminary and need to be followed up more carefully by examining potential cultural adaptations that may be acceptable to this population. For example, it may be useful to address perceptions of the usefulness of NRT and other pharmacologic treatments given that Hispanics/Latinos are less likely to use these products (Levinson et al., 2004). Likewise, because our results show stress is common and is perceived by Hispanic/Latino CWs as a determinant of their smoking, it may be useful to explore how stressors specific to this population (e.g., immigration- and workrelated) could be addressed in cessation interventions.

Other investigators measuring receptivity found mixed results. For instance, in a study of AfricanAmerican smokers, the researchers found equivocal results regarding a culturally specific intervention and intervention receptivity (Webb, Francis, Hines, \& Quarles, 2007). While some participants thought it could be useful, others were skeptical and worried the culturally specific intervention could fall to stereotype or have other negative components (Webb et al., 2007). Further, empirical support for culturally tailored behavioural interventions, including smoking cessations trials, have shown mixed levels of effectiveness (Nierkens et al., 2013). One recent study using a culturally tailored intervention among Latino smokers living with HIV/AIDS found the tailored intervention arm did not result in better cessation outcomes than the non-tailored (and less intensive) comparison intervention (Stanton et al., 2015). The investigators did not find any added benefit from the intervention, despite the support of tailored interventions in the literature (Stanton et al., 2015).

Finally, an emergent theme showed CWs were under a great deal of job stress which exacerbated their tendency to smoke at work. Stressful work environments are known to increase smoking, but the influence of these types of work environments on smoking cessation success are mixed (Albertsen, Borg, \& Oldenburg, 2006). For example, in a systematic review of how work environments 
impact smoking cessation, researchers found higher stress jobs were associated with higher smoking rates; however, they also found the availability of cessation resources at work increased the probability of smoking cessation (Albertsen et al., 2006).

The data need to be interpreted with several limitations in mind. We only recruited male CWs from a large construction company operating on a number of sites. As such, we are unable to determine if women CWs have different preferences. Whereas women have made gains in construction trades, it remains overwhelmingly male dominated. In 1970, women comprised less than $1 \%$ of workers in construction trades, which only grew to $2.3 \%$ in 2,000 (CPWR - The Center for Construction Research and Training, 2013). Regardless, because CWs move from site to site, we are confident the male CWs in our sample represent a broader range of attitudes concerning the proposed cessation intervention. Additionally, we probed for participant receptivity for culturally adapting the cessation intervention, yet more rigorous work needs to be done to examine cultural tailoring in this population. Investigators need to design a more robust study and consider more factors than receptivity when creating culturally specific cessation interventions (Webb et al., 2007).

Additionally, our eligibility criteria called for selfreported current smokers, including light and intermittent smokers. The inclusion of the light/intermittent smokers was helpful to understand the needs of this important population sub-group of Hispanic/Latino smokers. However, even though we screened potential participants for current smoking status, our quantitative data revealed a possible non-smoker. Future studies should consider biochemically verifying smoking status, even for focus group participation. Despite the non-smoker in the group, we believe his presence did not alter the findings from the focus groups since it was only one participant out of 37. Further, because the data are qualitative, conclusions about the strength of our findings cannot be made (Mermelstein, 1999). Nevertheless, qualitative data allowed us to understand construction work environments and the target population's perceptions regarding the role of cigarettes in their lives. We also identified cessation barriers and developed an intervention best suited to their work locations. Hence, qualitative information provided us with unique data and valuable insight into how to create an intervention most useful to CWs.

Nevertheless, there are ways to mitigate some limitations and improve the rigour of qualitative research and data. One basic question to ask is "do the results have face validity" (Krueger, 1994)? To address this, we focused on data collection and analyses. To improve the rigour of our findings, we used five focus groups to obtain a robust dataset for analysis and stopped when we reached saturation (Mays \& Pope, 2000). Additionally, the primary investigator analysing the data discussed initial findings with focus group moderators to ensure s/he was capturing the concepts and themes for data interpretation (Kitto et al., 2008).

The main purpose of the study was to understand participant acceptability of a smoking cessation intervention that could possibly use the food truck, a resource on most construction sites, to help facilitate smoking cessation. This work provided insights into smoking cessation needs and preferences of Hispanic/Latino CWs, a large underserved population with high smoking rates. Finally, because university investigators are committed to a partnership with the construction firms and this population, as we obtain findings, we will disseminate them at meetings with the managers at participating construction sites.

\section{Financial Support}

This work was supported by the National Cancer Institute (NCI) at the National Institutes of Health (grant number R21 CA202993). NCI had no role in the study design, collection, or analysis, or interpretation of the data and manuscript writing. The content is solely the responsibility of the authors and does not necessarily represent the official views of the National Institutes of Health. Supplemental funding was provided by the University of Miami Sylvester Comprehensive Cancer Center.

\section{Conflict of Interest}

None.

\section{Ethical Standards}

The authors assert that all procedures contributing to this work comply with the ethical standards of the relevant national and institutional committees on human experimentation and with the Helsinki Declaration of 1975, as revised in 2008 .

\section{References}

Albertsen, K., Borg, V., \& Oldenburg, B. (2006). A systematic review of the impact of work environment on smoking cessation, relapse and amount smoked. Preventive Medicine, 43(4), 291-305. doi:10.1016/j.ypmed.2006.05.001.

American Cancer Society. (2016). Cancer Facts \& Figures 2016. Atlanta: American Cancer Society.

Boeije, H. (2002). A purposeful approach to the constant comparative method in the analysis of qualitative interviews. Quality \& Quantity, 36, 391-409.

Boulous, D. N. K., Loffredo, C. A., El Setouhy, M., Abdel-Aziz, F., Israel, E., \& Mohamed, M. K. (2009). Nondaily, light daily, and moderate-to-heavy cigarette smokers in a rual area of Egypt: A population-based survey. Nicotine and Tobacco Research, 11(2), 134-138.

Brown, A. (2014). U.S. Hispanic and Asian populations growing, but for different reasons. Retrieved from http://www.pewresearch.org/fact-tank/2014/06/26/u-shispanic-and-asian-populations-growing-but-fordifferent-reasons/. 
Caban-Martinez, A., Clarke, T., Davila, E., Fleming, L., \& Lee, D. (2011). Application of handheld devices to field research among underserved construction worker populations: A workplace health assessment pilot study. Environmental Health, 10(1), 27.

Centers for Disease Control and Prevention. (2016). Fact sheets: Tobacco related mortality. Retrieved from https://www.cdc.gov/tobacco/data_statistics/fact_sheets/ health_effects/tobacco_related_mortality/.

Corbin, J., \& Strauss, S. L. (2007). Basics of qualitative research: Grounded theory procedures and techniques (3rd ed.). London: Sage.

CPWR - The Center for Construction Research and Training. (2013). The construction chart book: The US construction industry and its workers. Silver Spring, MD. Retrieved from http://www.cpwr.com/sites/default/files/publications/ 5th\%20Edition\%20Chart\%20Book\%20Final.pdf.

Dong, S., Chowdhury, R., McCann, M., Trahan, C., \& Gittleman, J. (2002). The construction chart book: The U.S. Construction industry and its workers (3rd ed.). Silver Spring, MD: CPWR - Center for Construction Research and Training.

Driscoll, T., Steenland, K., Imel Nelson, D., \& Leigh, J. (2004). Occupational airborne particulates: Assessing the environmental burden of disease at national and local levels. Geneva: World Health Organization.

Driscoll, T., Steenland, K., Prüss-Ustun, A., Imel Nelson, D., \& Leigh, J. (2004). Occupational carcinogens: Assessing the environmental burden of disease at national and local levels. Geneva: World Health Organization.

Ennis, S. R., Rios-Vargas, M., \& Albert, N. G. (2011). The hispanic population: 2010. Retrieved from 2010 Census Briefs: http://www.census.gov/prod/cen2010/briefs/c2010br-04. pdf.

Glaser, B., \& Strauss, A. (1967). Discovery of grounded theory: Strategies for qualitative research. New York: Aldine De Gruyter.

Hawkins, R. P., Kreuter, M., Resnicow, K., Fishbein, M., \& Dijkstra, A. (2008). Understanding tailoring in communicating about health. Health Education Research, 23(3), 454466.

Hewitt-Taylor, J. (2001) Use of constant comparative analysis in qualitative research. Nursing Standard, 15(42), 39-42. Date of acceptance: March 192001.

Jamal, A., Homa, D. M., O’Connor, E., Babb, S. D., Caraballo, R. S., Singh, T. et al. (2015). Current cigarette smoking among adults - United States, 2005-2014. Morbidity and Mortality Weekly Report, 64(44), 1233-1240. doi:10.15585/mmwr.mm6444a2.

Jamal, A., King, B. A., Neff, L. J., Whitmill, J., Babb, S. D., \& Graffunder, C. M. (2016). Current cigarette smoking among adults - United States, 2005-2015. Morbidity and Mortality Weekly Report, 65(44), 1205-1211. doi:10.15585/mmwr.mm6544a2.

Kaplan, R. C., Bangdiwala, S. I., Barnhart, J. M., Castaneda, S. F., Gellman, M. D., Lee, D. J. et al. (2014). Smoking among U.S. Hispanic/Latino adults: The Hispanic community health study/study of Latinos. American Journal of Preventive Medicine, 46(5), 496-506. doi:10.1016/j.amepre.2014.01.014.

Kitto, S. C., Chesters, J., \& Grbich, C. (2008). Quality in qualitative research. The Medical Journal of Australia, 188(4), 243246. doi:kit10137_fm [pii].

Krueger, R. (1994). Focus groups: A practical guide for applied research, Second Edition ( Vol. Second Edition). Thousand Oaks, CA: Sage Publications.

Lee, D., Davila, E., LeBlanc, W., Caban-Martinez, A., Fleming, L., Christ, S. et al. ( October 2012). Morbidity and disability among workers 18 years and older in the Construction sector, 1997-2007 In (Vol. DHHS (NIOSH) Publication No. 2012-156. Department of Health and Human Services, Centers for Disease Control and Prevention, National Institute for Occupational Safety and Health.

Lee, D. J., Fleming, L. E., Arheart, K. L., LeBlanc, W. G., Caban, A. J., Chung-Bridges, K. et al. (2007). Smoking rate trends in U.S. occupational groups: The 1987 to 2004 National Health Interview Survey. Journal of Occupational and Environmental Medicine, 49(1), 75-81. doi:10.1097/JOM.0b013e31802ec68c.

Lee, D. J., Fleming, L. E., McCollister, K. E., Caban, A. J., Arheart, K. L., LeBlanc, W. G. et al. (2007). Healthcare provider smoking cessation advice among US worker groups. Tobacco Control, 16(5), 325-328. doi:10.1136/tc.2006.019117.

Levinson, A. H., Perez-Stable, E. J., Espinoza, P., Flores, E. T., \& Byers, T. E. (2004). Latinos report less use of pharmaceutical aids when trying to quit smoking. American Journal of Preventive Medicine, 26(2), 105-111.

Martinez-Tyson, D., Pathak, E. B., Soler-Vila, H., \& Flores, A. M. (2009). Looking under the Hispanic umbrella: Cancer mortality among Cubans, Mexicans, Puerto Ricans and other Hispanics in Florida. Journal of Immigrant and Minority Health, 11(4), 249-257. doi:10.1007/s10903-008-9152-4.

Mays, N., \& Pope, C. (2000). Qualitative research in health care. Assessing quality in qualitative research. British Medical Journal, 320(7226), 50-52.

Mermelstein, R. (1999). Explanations of ethnic and gender differences in youth smoking: A multi-site, qualitative investigation. The Tobacco Control Network Writing Group. Nicotine \& Tobacco Research, 1(Suppl. 1), S91-S98.

Merzel, C. R., Isasi, C. R., Strizich, G., Castaneda, S. F., Gellman, M., Maisonet Giachello, A. L. et al. (2015). Smoking cessation among U.S. Hispanic/Latino adults: Findings from the Hispanic Community Health Study/Study of Latinos (HCHS/SOL). Preventive Medicine, 81, 412-419. doi:10.1016/j.ypmed.2015.10.006.

Nierkens, V., Hartman, M. A., Nicolaou, M., Vissenberg, C., Beune, E. J., Hosper, K. et al. (2013). Effectiveness of cultural adaptations of interventions aimed at smoking cessation, diet, and/or physical activity in ethnic minorities. A systematic review. PLoS One, 8(10), e73373. doi:10.1371/journal.pone.0073373.

Pope, C., Ziebland, S., \& Mays, N. (2000). Qualitative research in health care: Analyzing qualitative data. BMJ, 320, 114-116, 2000 . 
Resnicow, K., Soler, R., Braithwaite, R. L., Ahluwalia, J. S., \& Butler, J. (2000). Cultural sensitivity in substance use prevention. Journal of Community Psychology, 28(3), 271-290.

Sorensen, G., Barbeau, E., Hunt, M. K., \& Emmons, K. (2004). Reducing social disparities in tobacco use: A socialcontextual model for reducing tobacco use among bluecollar workers. American Journal of Public Health, 2004/02/01, 94(2), 230-239.

Sorensen, G., Barbeau, E. M., Stoddard, A. M., Hunt, M. K., Goldman, R., Smith, A. et al. ( Feb 2007). Tools for health: The efficacy of a tailored intervention targeted for construction laborers. Cancer Causes \& Control: CCC, 18(1), 51-59.

Stanton, C. A., Papandonatos, G. D., Shuter, J., Bicki, A., LloydRichardson, E. E., de Dios, M. A. et al. (2015). Outcomes of a tailored intervention for cigarette smoking cessation among latinos living with HIV/AIDS. Nicotine \& Tobacco Research, 17(8), 975-982. doi:10.1093/ntr/ntv014.
Stead, L. F., Perera, R., Bullen, C., Mant, D., HartmannBoyce, J., Cahill, K. et al. (2012). Nicotine replacement therapy for smoking cessation. The Cochrane Database Systematic Reviews, 11, CD000146. doi:10.1002/14651858.CD000146.pub4.

Thomas, D. R. (2006). A general inductive approach for analyzing qualitative evaluation data american. Journal of Evaluation, 27(2), 237-246.

Webb, M. S., Francis, J., Hines, B. C., \& Quarles, F. B. (2007). Health disparities and culturally specific treatment: Perspectives and expectancies of African American smokers. Journal of Clinical Psycholology, 63(6), 567-583. doi:10.1002/jclp.20372.

Webb, M. S., Rodriguez-Esquivel, D., \& Baker, E. A. (2010). Smoking cessation interventions among Hispanics in the United States: A systematic review and mini meta-analysis. American Journal of Health Promotion, 25(2), 109-118. doi:10.4278/ajhp.090123-LIT-25. 